\title{
Multitemporal liver analysis for surgical planning and clinical follow-up, adapted for Sibgrapi 2019
}

\author{
Rodrigo Braga dos Santos*, Guilherme Fontes dos Reis*, Gilson Alexandre Ostwald Pedro da Costa* \\ *Department of Informatics and Computer Science \\ Rio de Janeiro State University (UERJ) \\ Rio de Janeiro, Brazil \\ Email: rbs758@mun.ca, fonte01@ cougars.csusm.edu, gilson.costa@ime.uerj.br
}

\begin{abstract}
Computed tomography (CT) images have been widely used in the diagnosis of diseases, in surgical planning and in transplantation preparation. Besides the identification and visualization of relevant structures, automatic tissue volume measurements, based on image segmentation are critical for computer-aided diagnosis and surgical planning [1], [2]. In particular, the visual analysis of liver images obtained by CT presents important challenges, mainly because the organ is characterized by a topographic anatomy which is different from its functional anatomy [3]. Currently, the common conditions for the analysis of computerized tomographies are still basically visual, thus performed through manual interpretation of $2 \mathrm{D}$ images [4]. According to [2], this ends up being an important source of errors, since the human expert is subjected to an exhaustive routine in the analysis of very large numbers of images, and fatigue can affect the interpretation process. In order to aid surgical planning and clinical follow-up, in this work we developed a web-base application that transforms CT images into 3D models, which can be used for computerized multitemporal analysis of livers.
\end{abstract}

\section{INTRODUCTION}

It is common knowledge that one of society's huge concerns is health. Cancer has being one of the biggest causes of death lately [5]. In this way advances, investments and efforts are focused on this disease with the aim of discovering ways and means to combat cancer and lower its impact on human health.

This work uses the liver as the object case of study. In this organ the types of cancers are divided into two categories: primary and secondary, or metastatic (originating in another organ that also reaches the liver). [5]

The diagnosis in liver cancer has a very short window of observation, where when detected, usually the patient already has the disease in an advanced stage. A tumor can double its size in just 4 months, compared to other types of tumors this is a very fast growing period. [6]

Therefore, early diagnosis is of paramount importance in liver cancer treatment process. Consequently, the development of examinations for diagnosis in a timely manner, so that appropriate treatment can be carried out depending on the stage of the disease, have been improved over the years. Additionally, non-invasive imaging tests represent the most modern tools for the early diagnosis of cancer. As examples, magnetic resonance imaging and computed tomography (CT) can be mentioned.
Computed tomography performed with dynamic intravenous contrast allows the recognition of liver neoplastic lesions with accuracy of $75 \%$ to $90 \%$. However, lesions smaller than 3 $\mathrm{cm}$ are harder to detect, because of their isodensity with the normal hepatic parenchyma [6].

Nonetheless, as the development of the imaging technology grows rapidly, even in advanced medical centers, the diagnosis process end up being primarily visual, starting from the analysis of a doctor, thus making it insufficiently fast and precise [2].

Although there are 3D exams, such as helical computed tomography, the analysis of the resulting images is still done in 2D [5]. There is, thus, a need for the development of diagnostic tools that allows 3D visualization, and multitemporal analysis, to aid surgical planning and clinical follow-up.

The present work falls within this scope in order to provide methods for the aid of multitemporal and surgical clinical follow-up, aiming at providing accurate information regarding the development of the disease, which can be obtained automatically. As a work in progress, so far an environment that supports the comparison of 3D models of the examinations of a patient carried out at different dates was developed. The environment makes it possible to analyze information regarding the evolution of the disease - the growth of liver size - allowing a professional to manipulate the 3D liver models in order to infer anomalies, reducing the analysis time and making it more precise, improving the way pathology analysis is performed.

\section{Methodology}

\section{D Model Synthesis}

In order to generate the three-dimensional liver, we first need to put the computed tomography into a previously proposed system, called 3DLiver. After loading the program we have to select one of the images in the tomography that contains the liver. Then, we need to draw a polygon so that the program can search for a liver based on this polygon using the level set method.

With the three-dimensional liver model generated, we use the VisIt program to convert the 3DLiver models' format from VTK to PLY. VisIt is an open source, interactive, scalable, visualization, animation and analysis tool. With it users can 
quickly generate visualizations, animate them in time, manipulate them with a variety of operators and mathematical expressions, and save the resulting images and animations for presentations. VisIt contains a rich set of visualization features to enable users to view a wide variety of data, including scalar and vector fields defined in two-dimensional and three-dimensional (2D and 3D) structured, adaptive, and unstructured meshes. With the three-dimensional PLY liver, we used our own program to soften it. The VTK class used to smooth the liver was vtkSmoothPolyDataFilter.

The vtkSmoothPolyDataFilter is a filter that adjusts point coordinates using Laplacian smoothing. The effect is to "relax" the mesh, making the cells better shaped and the vertices more evenly distributed.To achieve a best smoothing with the three-dimensional liver model the best parameters are with fifteen interactions, a relaxation factor, a smoothing feature and smoothing, and limit enabled. With these parameters it is possible to smooth the liver, as shown in Figure 1, making the three-dimensional object easier to visualize, and better resembling the human liver organ.

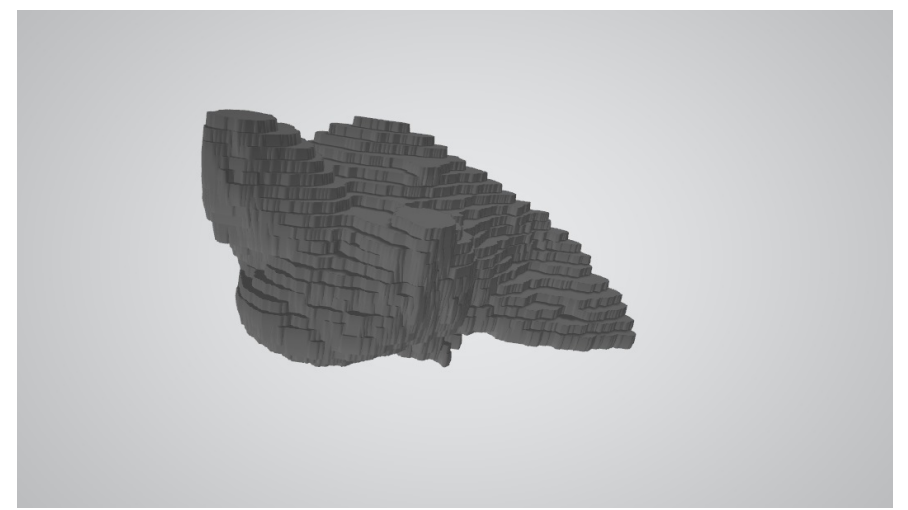

(a) Raw liver unprocessed

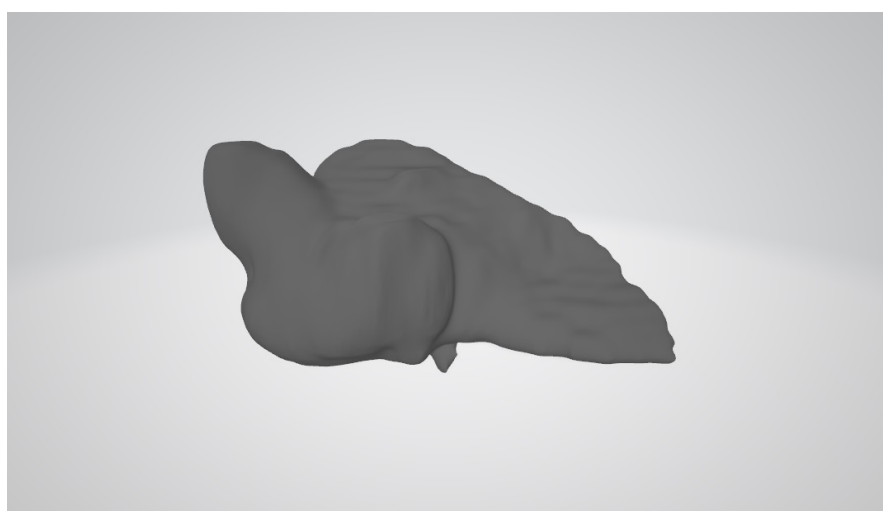

(b) Liver after filter is applied

Fig. 1. 3D model smoothing process

\section{Web Application}

For this project we have created a web application using PHP, MySQL, Javascript and others technologies in order to compare two 3D liver models. The environment has options to sign in as a Doctor or as a Patient. Doctors have an option to upload exams indexing it to patients registered on the application. Doctors can also compare exams uploaded. After selecting which exams of a specific patient the doctor can proceed with the analysis (see Figure 2).

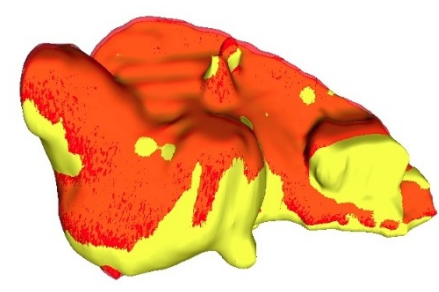

Fig. 2. Comparing overlapping livers.

Figure 2 shows the screen where one can display two liver models (of a same patient) and overlap them. In this picture, one 3D model has a yellowish color and the other red, so one can see where each one stands out and where the differences are. The volume of the $3 \mathrm{D}$ model is displayed at the top left and right. Also presented on the side are controllers for each 3D model, detailed in Figure 3. With the controller, one can move the model on the $\mathrm{X}, \mathrm{Y}$ and $\mathrm{Z}$ axes, as well as rotate around the different axes. An object scalability bar is displayed which can increase or decrease the size of the model.

Other features on this screen include the default button that moves the model to the starting position, the color selection buttons where you can change the color in which the 3D model is viewed, as well as the dropdown with the Flat Shading, Smooth Shading and Wireframe options, which correspond to the outline of the $3 \mathrm{D}$ model.

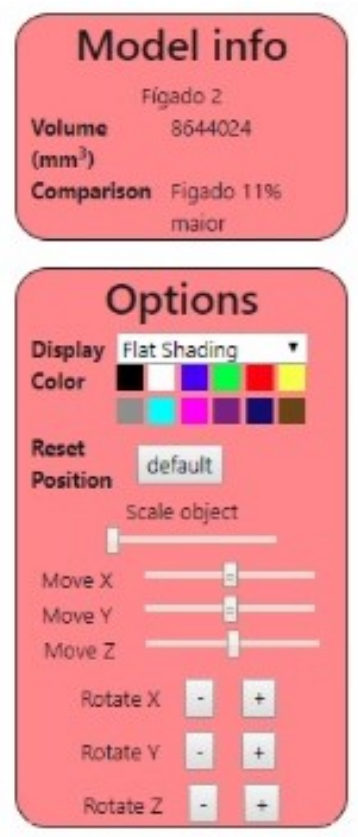

Fig. 3. 3D models control panel. 
In detail (Figure 3) we show the $3 D$ models control panel. There are 2 controls because the visualization is performed with 2 models, each control performs actions on the respective 3D model. In the control panel there are options to change liver color, to rotate it positively and negatively with respect to the axes. One can also scale the object by increasing it in size or shrinking it. The default button resets the model to the coordinates $\mathrm{X}=0, \mathrm{Y}=0, \mathrm{Z}=0$, so that overlapping of the models can occur again if the user has moved on some axis.

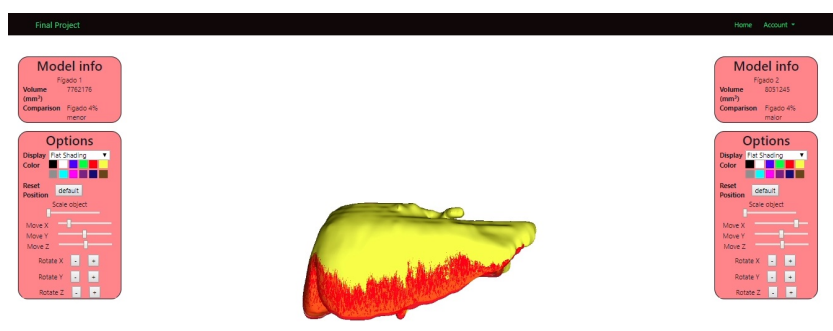

Fig. 4. Rotating models.

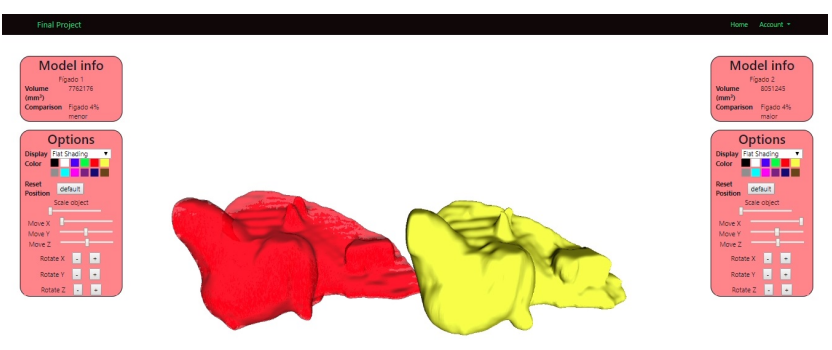

Fig. 5. Comparing livers side by side.

Figures 4 and 5 show some of the possibilities that the user with the controls. In Figure 4 it can be noticed that the models remain overlapping. On the other hand, in Figure 5 there was a movement in the $\mathrm{X}$ axes for both models.

\section{EXPERIMENTAL ANALYSIS}

Some experiments were designed to assess the gains in the diagnosis process and clinical follow-up with the tool developed in this work.

The Atlas of Cancer Genome and Hepatocellular Carcinoma of the Liver is a research community focusing on providing clinical data and images of patients with some type of cancer. Clinical, genetic and pathological data are in the Genomic Data Commons (GDC) Data Portal, while radiological data are stored in The Cancer Imaging Archive (TCIA).

In the ecxperiments we relied on radiological data from The Cancer Imaging Archive. The radiological data in the archive was obtained from all over the world, for the purpose of having a diversified database. In most cases, there is a sample of 500 radiological data per cancer type. For this reason, image data sets are extremely heterogeneous in terms of scanner modalities, manufacturers, and acquisition protocols. Also, in most cases, images were acquired as part of routine care and not as part of a controlled research study or clinical trial.
To perform a multi-temporal liver analysis, two CT scans of the same patient's abdominal region were chosen at different dates. The first being from 08/07/2001, with 88 images, and the second being from 17/08/2001, with 91 images. There was a difference of 71 days between the two exams.

Due to medical confidentiality it is not possible to know the identity of the patient. The patient information that the portal provides are gender, age, and additional data about the patient's medical history. The patient chosen was TCGA-DDA1EJ, identification number, discloses that the patient is a seventy-one years old female, with a history of malignant liver neoplasm [7].

In the sample exams used in this paper, it can be noted that the patient's liver grew $4 \%$ in a period of 71 days, which is consistent with the evolution of the disease in this patient.

\section{CONCLUSION}

This study focused on the difficulties involved in the production of a fast and precise diagnosis of liver cancer, and possibly other hepathic pathologies, from CT images.

Using the 3DLiver system, which generates 3D models from CT scans [5], we developed a tool that allows specialists to visualize and compare liver 3D models of a patient. The tool contains a set of functionalities for visualizing and manipulating individual models, and provides important size differentiation information. This allows the user to easily know how much the organ has grown/reduced during the analysis time-frame.

The tool developed in this work already includes several functionalities, especially visualization ones, that extend a previous work.

As a work in progress, we plan to include in the near future more functionalities in the developed tool, such as the option of computing different volumetric and area measures.

We also want to develop mechanisms to produce and visualize 3D models of the internal structures of livers, such as arteries and veins, and eventual tumors, in order to further facilitate diagnosis.

We also plan to enhance the experimental analysis, by comparing the automated results to those produced manually by experienced hepatologists from the University Hospital of Rio de Janeiro State University (HUPE).

\section{ACKNOWLEDGMENT}

The authors would like to thank Dr. Dário Augusto Borges Oliveira for supporting this work with technical data about the 3DLiver software.

We would also like to acknowledge the support from the Fundação de Amparo à Pesquisa do Estado do Rio de Janeiro (FAPERJ).

\section{REFERENCES}

[1] R. POHLE, "Segmentation of $3 \mathrm{~d}$ medical image data sets with a combination of region- based initial segmentation and active surfaces," 2003.

[2] L. F, "Lung cancers missed at low-dose helical ct screening in a general population: comparison of clinical, histopathologic, and imaging findings." 2002. 
[3] A. . P. J. COUINAUD, C.; DELMAS, Le foie: etudesanatomiques et chirurgicale. Masson, 1957.

[4] K. R, "Detection failures in spiral ct screening for lung cancer: analysis of ct findings." 1999.

[5] D. A. B. Oliveira, "Segmentação e visualização do fígado a partir de imagens de tomografia computadorizada," 2009.

[6] M. P. I. D. C. T. T. HEPÁTICAS, M ; CLÍNICAS, "Home artigos para o leigo câncer de fígado," 2008.

[7] S. K. e. a. Clark K, Vendt B, "The cancer imaging archive (tcia): Maintaining and operating a public information repository." 2013. 